\title{
Orbital autonomous inspection robot based on multi-sensor technology
}

\author{
Luo Hao ${ }^{1, a}$,Yan Biwu ${ }^{1, b}$,Zheng Lei ${ }^{1, c}$,Chen Hanchao ${ }^{1, d}$,Yin Pnegxiang ${ }^{1, e}$ \\ ${ }^{1}$ Wuhan NARI Limited Company of State Grid Electric Power Research Institute, in Wuhan, China; \\ a348379870@qq.com, ${ }^{b} 11318443 @ q q . c o m$, \\ c753134590@qq.com, ${ }^{\mathrm{d}} 396507281 @ q q . c o m,{ }^{\mathrm{e}} 1012425616 @ q q . c o m$
}

Keywords: inspection, robot, multi-sensor, autonomous, functions of sensor identification

Abstract. The overhead transmission line inspection robot takes the guide line as the working path. The obstacle operation is one of the online operation while inspecting along the line. In this paper, it set up a sensor network by classifying the obstacles on the overhead transmission lines. Before the robot perform obstacle, it uses of external sensors to identify the damper, suspension clamps and other lines on the various effects of inspection robot travels obstacles, determine the type of obstacles. Then perform the appropriate obstacle job, to achieve the robot autonomous patrol.

\section{Introduction}

It is an important and high frequency job for the maintenance unit of network operation to patrol the overhead transmission line, the traditional manual inspection work mode has already not satisfied the developing speed of power grid construction and the demand of the power apply reliability, to research and develop the independently patrol robot, which is adapt for the manual patrol that is not easy to carry out in the special geographical conditions $[1,2]$, has already become the research hot spot for the line operation and maintenance.

Inspection robot has taken the overhead transmission lines for orbit, it has installed the torsional damper、spacer、suspension clamp and other line hardware, this will block the inspection robot to walk straightly, so the robot may identify barriers and stride across the obstacles to complete line inspection task. So far, the inspection robot has two methods to identify barriers. One method is based on the prior knowledge of overhead transmission lines and GPS method of expert system $[3,4,5]$, this method utilizes the reasoning machine of expert system, and combines the static database from the prior knowledge of overhead transmission lines with the dynamic database from the GPS detects and the obtained real time information, to make a decision for identifying obstacles. This method is simple, as it has its defect or failure in the equipment on the line, such as, the torsional damper has its slip, at the same time, combined with the GPS positioning accuracy caused by the error, they will lead to the robot can't very good fix the position of the obstacles. The other method is a single sensor positioning. Through the robot vision to locate obstacles, however, as the influence of the background and lighting, detection accuracy is difficult to guarantee. The literature 8 fix the position of the obstacles by the ultrasonic sensors along the route, but it is limited by the sensor distance measuring range, thus it can only solve the problem of local positioning.

This article has many years of research and development, then it puts forward an obstacle positioning identification method for inspection robot, based on the more sensing technology, this method can accurately determine the distance and characteristics of the obstacle, thus it can assist the robot to go through the certain obstacle according to the characteristics of the obstacles, at last it realizes the inspection robot to independently patrol.

\section{Characteristics Analysis of Transmission Line obstacle}

As shown in Figure 1. The main obstacles which hinder the robot walking speed straight are suspension clamps, dampers, spacers and tension jumpers, while the inspection robot driving, crossing the obstacles, overhead the power transmission line. There are different forms of split spacer according to different voltage levels, but the split features similar to the suspension clamp. For the inspection 
robot, inspecting on the lower wire of the split wire, the pacer as an obstacle located is always above or aside the inspection rail.

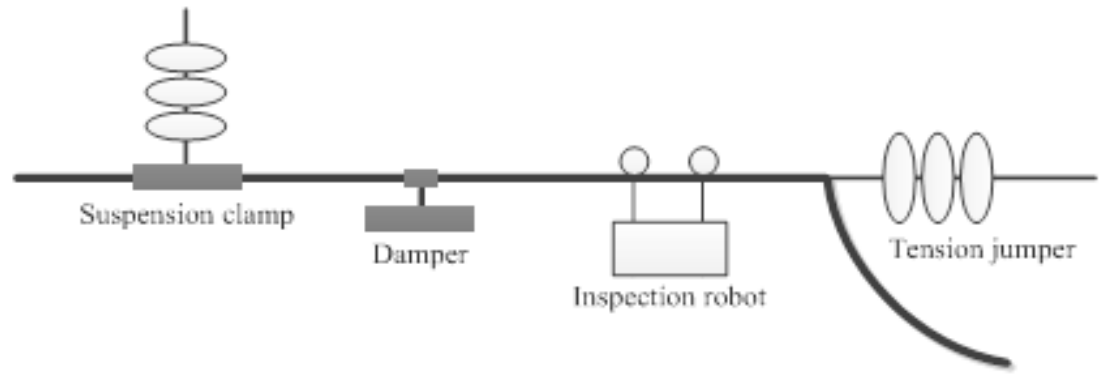

Figure 1 Obstacles on the power line for inspection robot

According to the structure characteristics, the installation status of the obstacle, combined with the walking method of the robot. The obstacles are classified into three which encountered during the robot inspecting. As shown in Table 1.

Table 1 classification of obstacles

\begin{tabular}{c|c|c}
\hline Classification of obstacle & Type & Characteristic \\
\hline Classification one & Dampers & Follow the wire \\
\hline Classification two & Spacers and & Above or aside the \\
& suspension clamps, & inspection wire \\
\hline Classification three & Tension jumpers & Arc track \\
\hline
\end{tabular}

\section{Obstacle detection method based on Multi-sensor}

To implement detection and automatic crossing the obstacle, in this paper, it installs multiple sensors to set up the sensor networks on the basis of size, structure, installation location of the obstacle on power lines.

(1)Obstacle Detection of Classification One

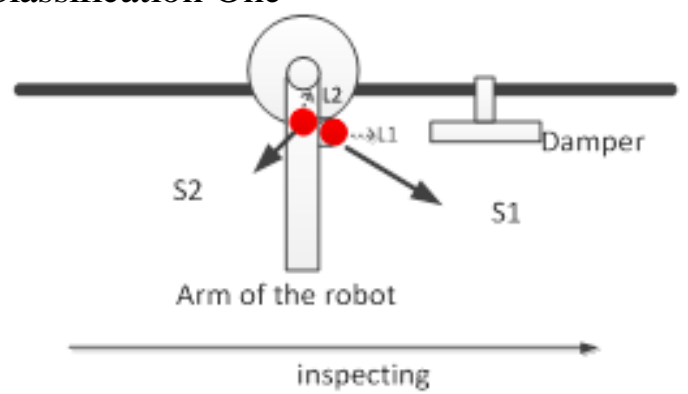

Figure 2 Obstacle detection schematic of Classification One

According to the characteristics of obstacle detection of classification one, set two photoelectric sensors (S1, S2) on the wheel ((As shown on Figure 2). S1 is located below the wire when robot inspects, used to detect the damper which is the obstacle of classification hanging on wires.

$\mathrm{S} 2$ is located below the wires, along the robot inspects direction, it emits the detecting perpendicular light to detecting the position of the wheel with the wire. The sensor outputs a digital signal. If there is no obstacles existing on the line, S1 emits lights without reflection, then the sensor outputs a low level signal (signal 0). When the inspection robot approaches the obstacles on the line, the sensor S1 receives the reflected signal, sensing the presence of an obstacle, then the sensor output a high level signal (signal 1).

Sensor S2 is mainly used to detect the wires, so the detection distance of S2 is adjusted $15 \mathrm{~mm}$ to prevent to detect other of the roller bodies and to ensure the uniqueness of the detecting results. S2 emits a light without reflection, when sensor outputs a low level signal (signal 0). 
Sensor S2 receives the reflected signals, senses the presence of an obstacle, it means that the wheel detaches from the wire, there are two situations this time:

1) The robot is crossing the obstacle, and the wheel detaches from the wire;

2) The robot has crossed the obstacle, ant the wheel return to the wire;

(2)Obstacle Detection of Classification Two

According to the characteristics of obstacle detection of classification two, set two photoelectric sensors (S1, S2) on the wheel (As shown on Figure 3).
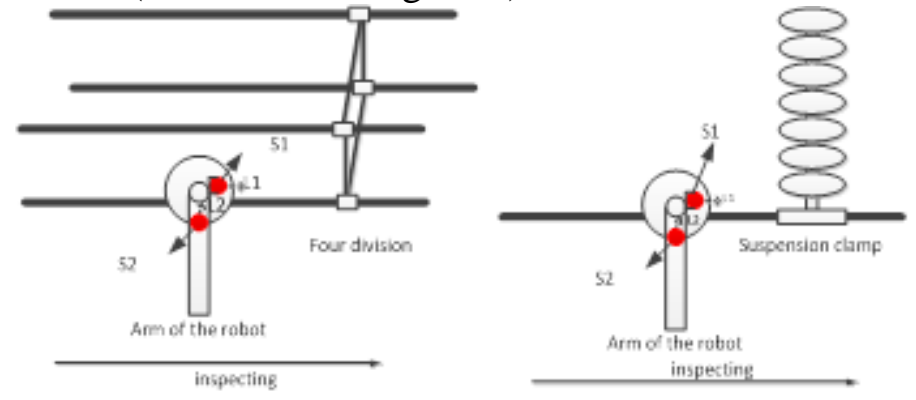

Figure 3 Detection Schematic of Four Division Spacer and Suspension Clamp

The Obstacle Detection of Classification two is the same principle as Classification two.

(3)Obstacle Detection of Classification Three

It can be judged on the basis of this feature that the obstacle of classification three including classification one and classification two. As shown on Figure 4, installed sensors S1, S2, S3, S4 on the wheel. S3 is a positioning sensor for the wire to determine the position of the wheel structure and wire, provides information on the wheel returning to the wire. S1, S2, S4 are positioning sensors of obstacles.S1 and S2, or S1 and S4 are detected an obstacle at the same time, that means the obstacle belonged to classification three.

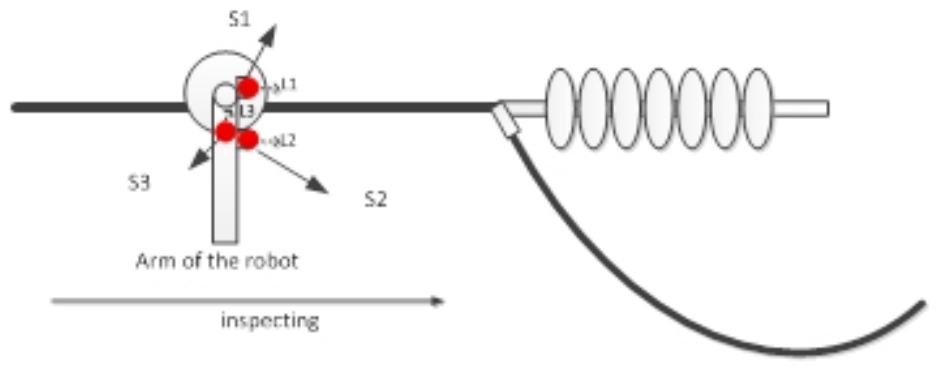

Figure 4 Obstacle detection schematic of Classification Five

\section{Sensor network set up to identify obstacles}

Through various detection schemes, Set up the sensor networks of the inspection robot. Requirements of the sensor networking are as follows:

1) Detection range covers all Classifications of obstacles;

2) Meet bi-detecting requirements;

3) Achieve the detection with minimal sensors;

For the requirements of sensor networking, obstacle detection sensor network of inspection robot was set up as follows:

1) Installs sensors which can detect all the obstacle classification in right place on inspection robot's arms,

2) Installs sensors both before and after of the inspection robot's arm ,to achieve obstacle detection bidirectional.

The sensor network on the one of the inspection robot arms, as shown in Figure 5.

The feature of classification three including classification one and classification two, so it can identify the obstacles through a combination of sensor information. The inspection robot identifies the obstacles based on the sensor signal, as shown in Table 2. 


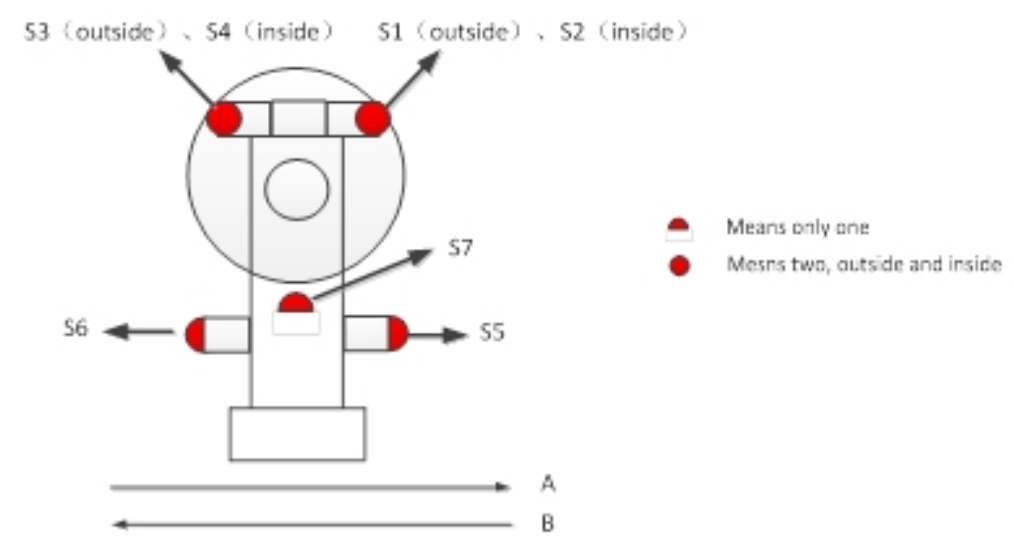

Figure 5 Sensor Network schematic

Table 2 Inspection robot identifies the obstacles based on the sensor signal

\begin{tabular}{c|c|c|c|c|c|c|c}
\hline Sensors & S1 & S2 & S3 & S4 & S5 & S6 & S7 \\
\hline \multirow{2}{*}{ Classification One(Direction A) } & 1 & 0 & 0 & 0 & 0 & 0 & 0 \\
\cline { 2 - 8 } & 0 & 1 & 0 & 0 & 0 & 0 & 0 \\
\hline Classification Two(Direction A) & 0 & 0 & 0 & 0 & 1 & 0 & 0 \\
\hline \multirow{2}{*}{ Classification One(Direction B) } & 0 & 0 & 0 & 1 & 0 & 0 & 0 \\
\cline { 2 - 8 } & 0 & 0 & 1 & 0 & 0 & 0 & 0 \\
\hline Classification Two(Direction B) & 0 & 0 & 0 & 0 & 0 & 1 & 0 \\
\hline \multirow{2}{*}{ Classification Three(Direction A) } & 1 & $0(1)$ & 0 & 0 & 1 & 0 & 0 \\
\cline { 2 - 8 } & 0 & 1 & 0 & 0 & 1 & 0 & 0 \\
\hline \multirow{2}{*}{ Classification Three(Direction B) } & 0 & 0 & 1 & $0(1)$ & 0 & 0 & \\
\cline { 2 - 8 } & 0 & 0 & 0 & 1 & 0 & 0 & 0 \\
\hline Detection of Wire & 0 & 0 & 0 & 0 & 0 & 0 & 1 \\
\hline
\end{tabular}

\section{Experiments}

Sensors are installed on inspection robot, as shown in Figure 6.There are three sensors on the robot arm unilaterally. Sensor 1 and sensor 2 form a certain angle that obstacle detecting of without dead ends. The sensor 3 detects the obstacle below the wire.
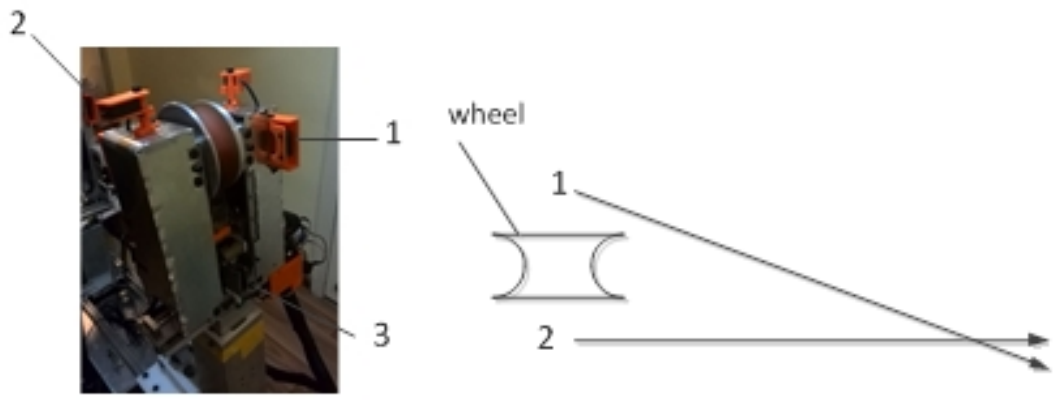

Figure 6 Sensor Network of Inspection Robot

Completed the sensor network of inspection robot, and then tests the reliability of the network, as shown in figure 7. Experiments are carried out five times for classification one and classification two. The results are shown in Table 3. 

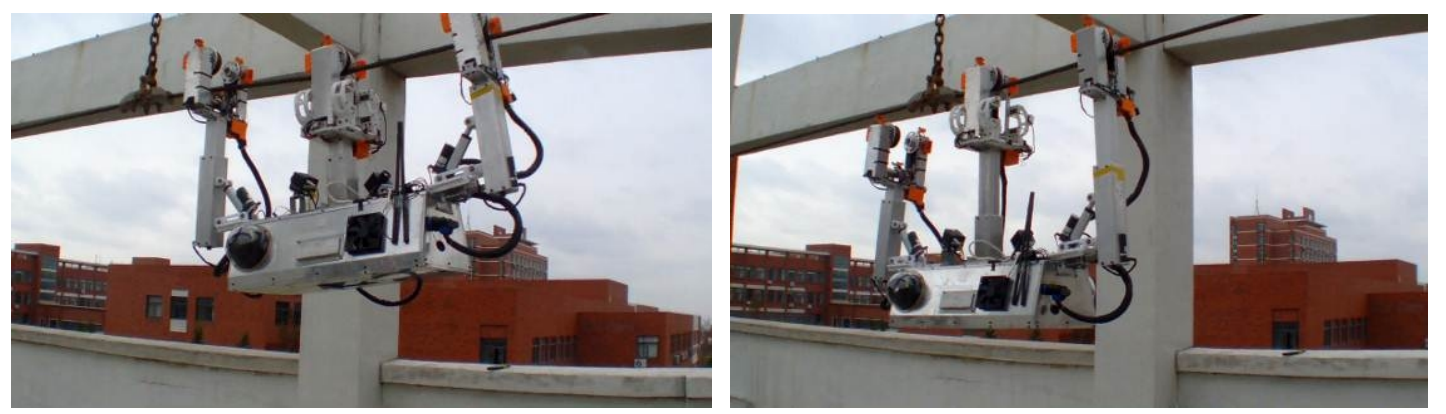

Figure 7 Testing the reliability of the network

Table 3 Results of Experiments for classification one and classification two

\begin{tabular}{c|c|c|c}
\hline sensors & 1 & 2 & 3 \\
\hline suspension clamps & 10 & 10 & 0 \\
\hline dampers & 0 & 0 & 10 \\
\hline
\end{tabular}

Experimental results show that, this kind of installation can accurately detect the obstacles of classification one and classification two and there are just three unilaterally. Therefore this program may be used in all the arms of the inspection robot.

\section{Summary}

It summarizes the characteristics of the obstacles, studied by obstacle characteristics of the transmission line inspection robot on line. According to the characteristics of the obstacle, the obstacles are classified into three, which clear the technology requirements of sensor network for obstacle recognition.According to the characteristics, set up the obstacle sensor networks, and experiments to achieve the inspection robot automatically detecting the type of obstacle and identifying the type of obstacle.

\section{References}

[1] Wu Gongping, Xiao Xiaohui, Xiao Hua, ect. Overhead High Voltage Transmission Line Inspection Robot Prototype[J]. Automation of Electric Power Systems. 2006,30 (13):97-107.

[2] Montambault S, Poulint N, Design and validation of a mobil robot for the power line inspection and maintenance[C],International Conference on Field and Service Robotics-FSR2007,Chamonix,FrancelSpringer,2008:495-504.

[3] Tang Li,Fang Lijin,Wang Hongguang, ect. Based on Distributed Expert System for EHV Transmission Line Inspection Robot Control System[J],Robots,2008,26(3):267-271.

[4] Zhou Fengyu,Wu Aiguo,Li Yibin. 110kv Transmission Line Inspection Robot Control Method and Implementation[J]. EDUCATION of ShanDong University.2007,37(6):31-35.

[5] Li Qiang. Obstacle transmission line inspection robot servo control based on multi-sensor[J]. Electric Automation.2009,31(1):9-12. 